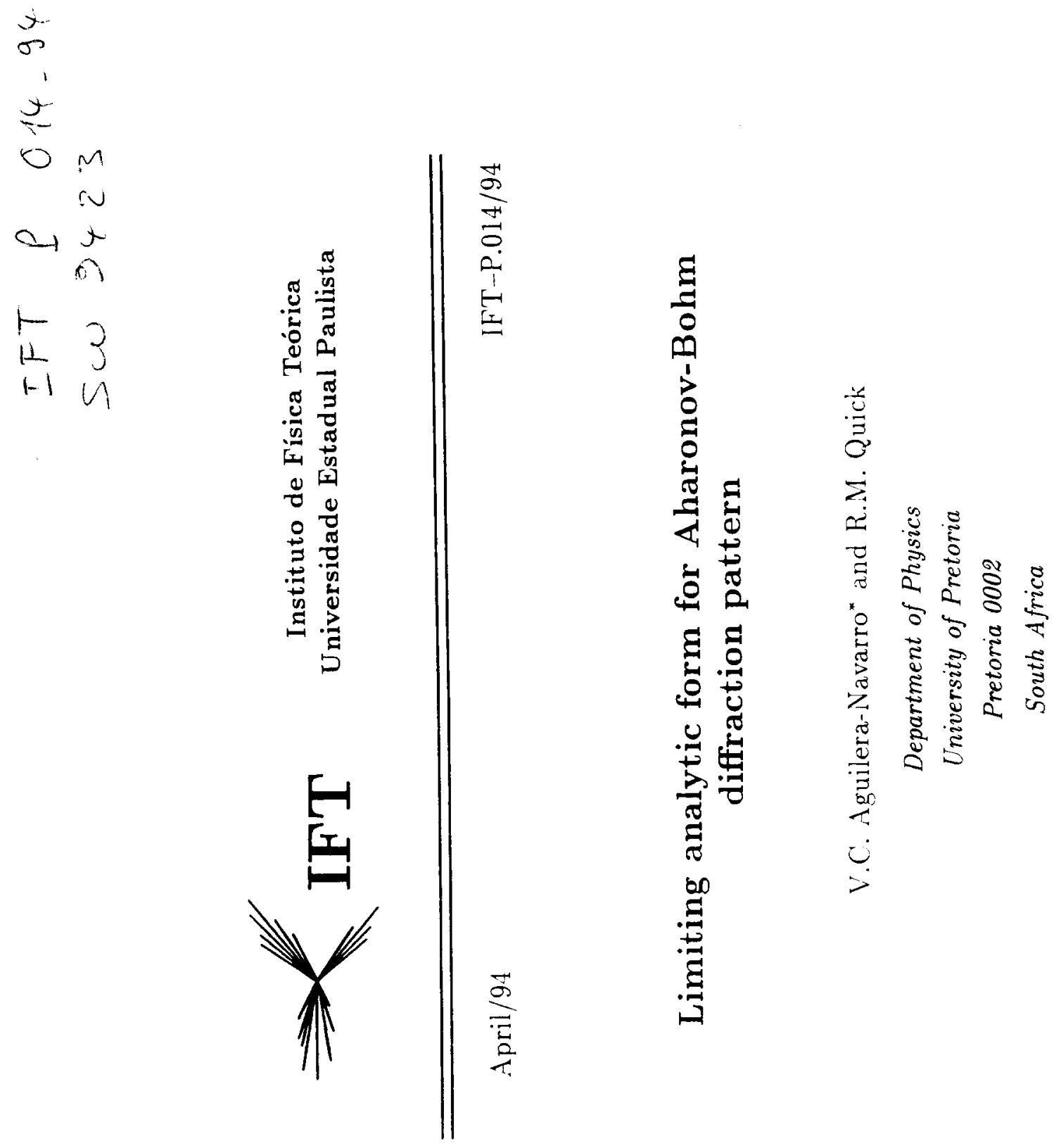




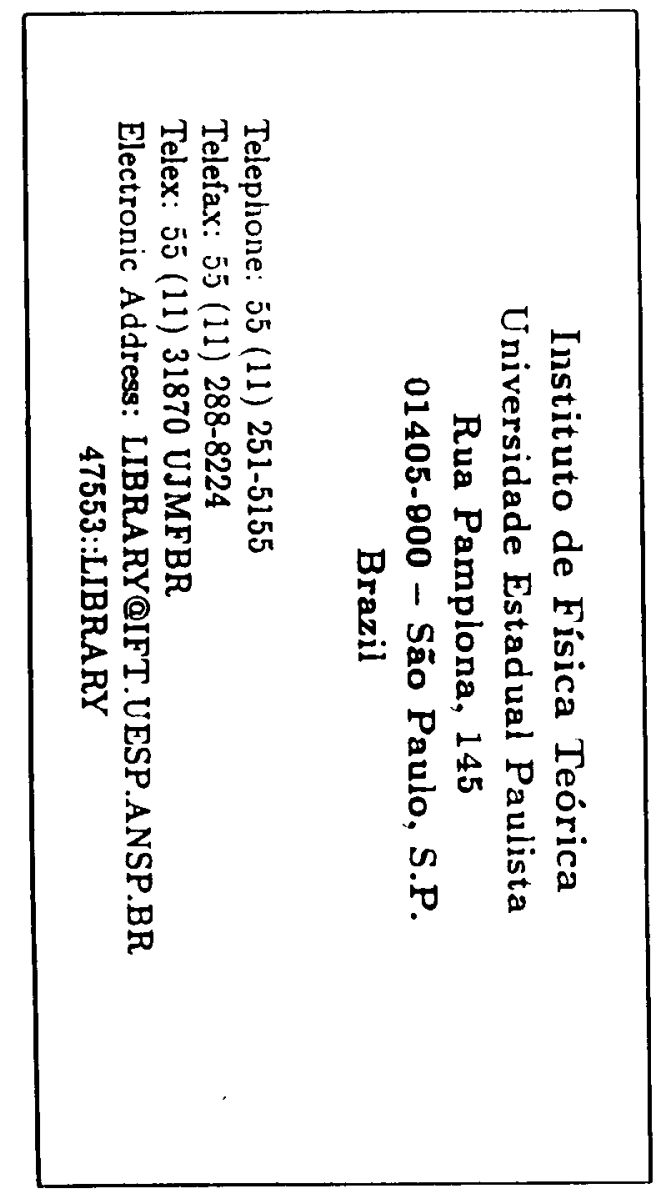




\section{Limiting analytic form for Aharonov-Bohm diffraction pattern}

V.C. Aguilera-Navarro * and R.M. Quick

Department of Physics, University of Pretoria, Pretoria, 0002, South Africa

$$
\text { (April 13, 1994) }
$$

\section{Abstract}

We consider a two-slit diffraction experiment with a magnetic flux confined to an inaccessible region between two rectangular slits. We then obtain a leading order analytic form for the asymmetry in the resulting diffraction pattern. The corrections to the expression are bounded and disappear in the limit of long wavelengths and/or infinite source-slit-screen spacing. Using the analytic form obtained, we obtain a non-zero value for the asymmetry in the number of electrons scattered to the left and to the right but a zero value for their average displacement.

03.65.Bz, 03.65. Nk

*On leave of absence from Instituto de Física Teórica, São Paulo, SP - Brazil
When a beam of electrons passes through two slits, the two resultant beams superpose afterwards producing a well known diffraction pattern. If additionally, a magnetic flux is confined to an inaccessible region between the two slits, then the diffraction pattern is shifted by an amount depending on the magnitude of the flux. This occurs despite the fact that the flux is "invisible" to the electrons. This unexpected result is known as the Aharonov-Bohm effect, after Y. Aharonov and D. Bohm [1].

The shift in the diffraction pattern has been confirmed experimentally [2]. However, there remain some unsolved problems related to the moments of the position at the observation screen. In particular, the first moment, i.e., the expectation value of the position of the electrons arriving at the observation screen, has been the subject of theoretical analyses with contradictory conclusions. The question is: Is the expected displacement zero or not ? Several authors [3]- [8] clajm that the expected displacement is zero for all values of the flux. On the other hand, explicit calculations based on Feynman path-integral technique show that that value is not zero, in general $[9,10]$. Unfortunately, the answer to the question cannot be formulated on the basis of general principles only. Ehrenfest's theorem cannot be invoked to show that the expectation value vanishes since there is no violation of this theorem if the expected displacement is non-zero [10]. The no-shift theorem of Semon and Taylor [6]. [8] cannot be applied since it is not clear if the theorem is true in general [11].

In the following we readdress this question. In Section II, we consider a delta function to describe the initial wavefunction, i.e., the electron source, and apply the Feynman path-integral method [12] to determine the wavefunction at the observation screen. The diffraction pattern is obtained as well as the asymmetry in the distribution of the arriving electrons scattered by the two slits. An analytical form for the leading contribution to this asymmetry is obtained, and a bound to the corrections is determined. In Section III, we evaluate the asymmetry parameter (the number of electrons scattered to the right minus the number of those scattered to the left) and the expected displacement. We find that the 
expected displacement is bounded by a bound which approaches zero in the limit of infinite wavelengths and/or infinite system size. This suggests but does not prove that it should be always zero. The bound also contradicts the numerical results obtained earlier in reference [10] and can be used as a test on any numerical calculation. It is not possible however to analyse the results obtained earlier by Kobe [9] since he employed Gaussian slits which are overlapping. Conclusions are drawn in Section IV.

\section{DIFFRACTION PATTERNS}

The Feynman path integral approach to quantum mechanics $\{4,5,9,12\}$ has been used to calculate the single-slit wavefunction. Then the two-slit wavefunction is given as the linear superposition of the wavefunctions from the two slits. The diffraction pattern is proportional to the square of the modulus of the wavefunction.

\section{A. Single-slit diffraction pattern}

We consider here the model introduced by Kobe [9]. According to this model the motion perpendicular to the screens (see Fig. 1) is classical while the motion parallel to the screens is to be treated quantum mechanically. This approximation was also used by Kobe et al. [10] and implicitly by Shapiro and Henneberger [13].

The approximation is justified since the action in the $y$ direction, $S_{y}$, is many orders of magnitude greater than $\hbar$. The action is the average kinetic energy in the $y$ direction i.e. $S_{y}=m \overline{v_{y}^{2}} / 2$ where $m$ is the mass of the electron, and $\overline{v_{y}^{2}} \sim \bar{v}_{y}^{2}=\left(y_{c}-y_{a}\right)^{2} /\left(t_{c}-t_{a}\right)^{2}$ is the average of the square of the velocity in the $y$ direction in going from the space-time point $\left(y_{a}, t_{a}\right)$ to $\left(y_{c}, t_{c}\right)$. Therefore $S_{y} / \hbar=(m / 2 \hbar) \bar{v}_{y} L \sim(L+l) / \lambda$ where $L+l=y_{c}-y_{a}$ and the reduced de Broglie wavelength is $\lambda=\hbar / m \bar{v}_{y}$. For realistic experimental setups this ratio is in fact very large e.g. for the parameters of the experiment by Jönsson [14] with $\lambda=10^{-6} \mu \mathrm{m}$ and with reasonable distance $L+l=11 \mathrm{~m}$, one has $S_{y} / \hbar \sim 10^{12}$
Thus the path integral in the $y$ direction can be evaluated in the saddle point approximation so that the electron only follows the classical path in this direction. We thus have a complete path integral formulation starting from the source and ending at the screens but because $S_{y}>>\hbar$ one can eliminate the path integral in the $y$ direction and replace $y$ by its classical value i.e.

$$
y-y_{a}=v\left(t-t_{a}\right)
$$

where $v \equiv \bar{v}_{y}$ denotes the (constant) velocity in the $y$ direction. For the single-slit geometry shown in Figure 1, the wavefunction at the observation screen is given by

$$
\Psi_{+}\left(x_{c}\right)=\int_{-\infty}^{\infty} d x_{b} \int_{-\infty}^{\infty} d x_{a} K^{0}\left(x_{c}, t_{c} ; x_{b}, t_{b}\right) G_{+}\left(x_{b}\right) K^{0}\left(x_{b}, t_{b} ; x_{a}, t_{a}\right) \Psi_{a}\left(x_{a}\right)
$$

where $G_{+}\left(x_{b}\right)$ is the transmission function for a single rectangular slit of width $2 b$ centered at $x_{0}$ :

$$
G_{+}\left(x_{b}\right)=\left\{\begin{array}{l}
0 \text { if }\left|x_{b}-x_{0}\right|>b \\
1 \text { if }\left|x_{b}-x_{0}\right|<b
\end{array}\right.
$$

We have taken the initial wave packet to be a delta function, as in the calculation of Kobe [9], and Shapiro and Henneberger [13]

$$
\Psi_{a}\left(x_{a}\right)=\delta\left(x_{a}\right)
$$

The free propagator is given by [12]

$$
K^{0}\left(x_{c}, t_{c} ; x_{b}, t_{b}\right)=\left[\frac{m}{i h\left(t_{c}-t_{b}\right)}\right]^{\frac{1}{2}} \exp \left[\frac{i m \pi\left(x_{c}-x_{b}\right)^{2}}{h\left(t_{c}-t_{b}\right)}\right],
$$

where $m$ is the mass of the electron and $h$ is Planck's constant. A similar formula holds for $K^{0}\left(x_{b}, t_{b} ; x_{a}, t_{a}\right)$. As shown in Fig. 1 , let $l$ denote the distance from the source to the screen with the slits; then

$$
l \equiv y_{b}-y_{a}=v\left(t_{b}-t_{a}\right)
$$

and let $L$ denote the distance between the screen with the slits and the observation screen then 


$$
L \equiv y_{c}-y_{b}=v\left(t_{c}-t_{b}\right)
$$

Substituting equations (2.3-2.5) and definitions (2.6-2.7) into (2.2) and performing the integrations yields

$$
\Psi_{+}\left(x_{\mathrm{c}}\right)=\frac{1}{i} N \mathrm{e}^{\frac{i m \pi}{h} \frac{e_{c}^{2}}{l_{c}}}\left[E i\left(\beta\left(x_{0}+b-\frac{l}{l+L} x_{c}\right)\right)-E i\left(\beta\left(x_{0}-b-\frac{l}{l+L} x_{c}\right)\right)\right]
$$

where the constants are given by

$$
N=\sqrt{\frac{1}{2 \lambda(l+L)}}
$$

and

$$
\beta=\sqrt{\frac{2}{\lambda}\left(\frac{1}{l}+\frac{1}{L}\right)}
$$

where $\lambda$ is the de Broglie wavelength of the electron

$$
\lambda=\frac{h}{m v}
$$

and $E i(z)$ denotes the complex Fresnel integral defined by

$$
E i(z) \equiv \int_{0}^{z} d \eta e^{i \frac{\pi}{2} \eta^{2}}
$$

B. Two-slit diffraction pattern

The geometry for a two-slit experiment is as illustrated in Figure 2 and the total wavefunction is given by superposing the wavefunctions from the two slits. Note that the use of the path-integral formalism ensures that the quantum mechanical treatment starts at the origin of the particles and not at the slits which ensures the coherence of the waves at each of the slits. In the absence of any magnetic flux the wavefunction at the observation screen is given by

$$
\Psi\left(x_{c}\right)=\Psi_{+}\left(x_{c}\right)+\Psi_{-}\left(x_{c}\right)
$$

where $\Psi_{+}\left(x_{c}\right)$ is the wavefunction from the slit centered at $x_{0}(2.8)$ and $\Psi_{-}\left(x_{c}\right)$ is the wavefunction from the slit centered at $-x_{0}$. The wavefunction $\Psi_{-}\left(x_{c}\right)$ is obtained by simply replacing $x_{0}$ by $-x_{0}$ everywhere in (2.8). From equation (2.2) and the relationship $G_{+}\left(x_{b}\right)=$ $G_{-}\left(-x_{b}\right)$ it can be seen that

$$
\Psi_{--}\left(x_{c}\right)=\Psi_{+}\left(-x_{c}\right)
$$

If a shielded magnetic flux is present then the wavefunctions for the two slits acquire an additional phase factor [1]

$$
\Psi_{ \pm}^{\prime}\left(x_{c}\right)=\exp \left(\frac{i q}{\hbar c} \oint_{C_{ \pm}} \mathbf{A} \cdot \mathbf{d r}\right) \Psi_{ \pm}\left(x_{c}\right)
$$

where $q$ is the charge of the particle, $\mathbf{A}$ is the magnetic vector potential and $C_{+}\left(C_{-}\right)$is the path from $x_{a}, t_{a}$ to $x_{c}, t_{c}$ via the slit at $x_{0}\left(-x_{0}\right)$. The total wavefunction at the observation screen is then

$$
\begin{aligned}
\Psi^{\prime}\left(x_{c}\right) & =\Psi_{+}^{\prime}\left(x_{c}\right)+\Psi_{-}^{\prime}\left(x_{c}\right) \\
& \left.=\exp \left(\frac{i q}{\hbar c} \int_{C_{+}} \mathbf{A} \cdot d \mathbf{r}\right)\left[\Psi_{+}\left(x_{c}\right)+\mathrm{e}^{-i \phi} \Psi_{-}\left(x_{c}\right)\right]\right)
\end{aligned}
$$

where $\phi=\frac{q \Phi}{\hbar c}$ is the magnetic flux parameter and

$$
\Phi \equiv \int_{S} \mathbf{B} \cdot d \alpha=\oint_{C} \mathbf{A} \cdot d \mathbf{r}
$$

is the enclosed magnetic flux. The diffraction pattern is then proportional to the magnitude of the wavefunction squared i.e.

$$
\left|\Psi^{\prime}\left(x_{c}\right)\right|^{2}=\left|\Psi_{+}\left(x_{c}\right)\right|^{2}+\left|\Psi_{--}\left(x_{c}\right)\right|^{2}+2 \cos \phi R e\left[\Psi_{+}^{*}\left(x_{c}\right) \Psi_{-}\left(x_{c}\right)\right]+2 \sin \phi \operatorname{lm}\left[\Psi_{+}^{*}\left(x_{c}\right) \Psi_{-}\left(x_{c}\right)\right]
$$

\section{Asymmetry in two-slit diffraction patter"}

The asymmetry in the two-slit diffraction pattern defined by 


$$
A\left(x_{c}\right) \equiv\left|\Psi^{\prime}\left(x_{c}\right)\right|^{2}-\left|\Psi^{\prime}\left(--x_{c}\right)\right|^{2}
$$

can be evaluated as

$$
A\left(x_{c}\right)=4 \sin \phi \operatorname{Im}\left[\Psi_{+}^{*}\left(x_{c}\right) \Psi_{-}\left(x_{c}\right)\right]
$$

Substituting the analytic expressions for $\Psi_{+}\left(x_{c}\right)(2.8)$ and $\Psi_{-}\left(x_{c}\right)=\Psi_{+}\left(-x_{c}\right)$ one obtains

$$
\begin{aligned}
A\left(x_{c}\right)=4 \sin \phi N^{2} & {\left[\left(C\left(\beta\left[x_{0}+b-\frac{l}{L+l} x_{c}\right]\right)-C\left(\beta\left[x_{0}-b-\frac{l}{L+l} x_{c}\right]\right)\right)\right.} \\
& \left.\left(S \mid \beta\left(x_{0}+b+\frac{l}{L+l} x_{c}\right]\right)-S\left(\beta\left[x_{0}-b+\frac{l}{L+l} x_{c}\right]\right)\right) \\
& \left.\left.\left(S\left(\beta \mid x_{0}+b-\frac{l}{L+l} x_{c}\right]\right)-S\left(\beta \mid x_{0}-b-\frac{l}{L+l} x_{c}\right]\right)\right) \\
& \left.\left.\left(C\left(\beta \mid x_{0}+b+\frac{l}{L+l} x_{c}\right]\right)-C\left(\beta\left[x_{0}-b+\frac{l}{L+l} x_{c}\right]\right)\right)\right]
\end{aligned}
$$

where $N$ and $\beta$ denote the constants given in equations (2.9) and (2.10) respectively, and $C(z)$ and $S(z)$ denote the cosine and sine Fresnel integrals

$$
\begin{aligned}
C(z) & \equiv \int_{0}^{z} \cos \left(\frac{\pi}{2} \eta^{2}\right) d \eta \\
S(z) & \equiv \int_{0}^{z} \sin \left(\frac{\pi}{2} \eta^{2}\right) d \eta
\end{aligned}
$$

Defining the following dimensionless constants

$$
\begin{aligned}
B & \equiv \beta b \\
X_{0} & \equiv \beta x_{0} \\
X_{c} & \equiv \beta \frac{l}{l+L} x_{c}
\end{aligned}
$$

the expression (2.21) becomes

$$
\begin{aligned}
A\left(x_{c}\right)=4 \sin \phi N^{2}[ & \int_{X_{0}-X_{c}-B}^{X_{0}-X_{c}+B} d \eta_{1} \cos \left(\frac{\pi}{2} \eta_{1}^{2}\right) \int_{X_{0}+X_{c}-B}^{X_{0}+X_{c}+B} d \eta_{2} \sin \left(\frac{\pi}{2} \eta_{2}^{2}\right)- \\
& \left.\int_{X_{0}-X_{c}-B}^{X_{0}-X_{c}+B} d \eta_{1} \sin \left(\frac{\pi}{2} \eta_{1}^{2}\right) \int_{X_{0}+X_{c}-B}^{X_{0}+X_{c}+B} d \eta_{2} \cos \left(\frac{\pi}{2} \eta_{2}^{2}\right)\right]
\end{aligned}
$$

We then redefine the variables of integration so that all the integrals run from $-B$ to $B$ to obtain

$$
A\left(x_{c}\right)=4 \sin \phi N^{2}\left[I_{1} \cos \left(2 \pi X_{c} X_{0}\right)+I_{2} \sin \left(2 \pi X_{c} X_{0}\right)\right]
$$

where

$$
\begin{aligned}
I_{1}= & 2 \int_{-B}^{B} d \eta_{1} \int_{-B}^{B} d \eta_{2} \sin \left(\pi \eta_{1} X_{c}\right) \sin \left(\pi \eta_{1} X_{0}\right) \cos \left(\pi \eta_{2} X_{c}\right) \cos \left(\pi \eta_{2} X_{0}\right) \\
& \sin \left(\frac{\pi}{2}\left(\eta_{2}^{2}-\eta_{1}^{2}\right)\right) \\
I_{2}= & \int_{-B}^{B} d \eta_{1} \int_{-B}^{B} d \eta_{2}\left\{\cos \left(\pi \eta_{1} X_{c}\right) \cos \left(\pi \eta_{1} X_{0}\right) \cos \left(\pi \eta_{2} X_{c}\right) \cos \left(\pi \eta_{2} X_{0}\right)\right. \\
- & \left.\sin \left(\pi \eta_{1} X_{c}\right) \sin \left(\pi \eta_{1} X_{0}\right) \sin \left(\pi \eta_{2} X_{c}\right) \sin \left(\pi \eta_{2} X_{0}\right)\right\} \cos \left(\frac{\pi}{2}\left(\eta_{2}^{2}-\eta_{1}^{2}\right)\right)
\end{aligned}
$$

Splitting $I_{2}$ into three parts yields

$$
\begin{aligned}
I_{2} & =\int_{-B}^{B} d \eta_{1} \int_{-B}^{B} d \eta_{2} \cos \left(\pi \eta_{1} X_{c}\right) \cos \left(\pi \eta_{2} X_{c}\right)+I_{3}-I_{4} \\
& =\frac{4}{\pi^{2} X_{c}^{2}} \sin ^{2}\left(\pi B X_{c}\right)+I_{3}-I_{4} \\
I_{3} & =\int_{-B}^{B} d \eta_{1} \int_{-B}^{B} d \eta_{2} \cos \left(\pi \eta_{1} X_{c}\right) \cos \left(\pi \eta_{2} X_{c}\right)\left[\cos \left(\pi \eta_{1} X_{0}\right) \cos \left(\pi \eta_{2} X_{0}\right) \cos \left(\frac{\pi}{2}\left(\eta_{2}^{2}-\eta_{1}^{2}\right)\right)-1\right] \\
I_{4} & =\int_{-B}^{B} d \eta_{1} \int_{-B}^{B} d \eta_{2} \sin \left(\pi \eta_{1} X_{c}\right) \sin \left(\pi \eta_{2} X_{c}\right) \sin \left(\pi \eta_{1} X_{0}\right) \sin \left(\pi \eta_{2} X_{0}\right) \cos \left(\frac{\pi}{2}\left(\eta_{2}^{2}-\eta_{1}^{2}\right)\right)
\end{aligned}
$$

Finally therefore we obtain the asymmetry $(2.19)$ in the form

$$
A\left(x_{c}\right)=4 \sin \phi N^{2}\left[\frac{4}{\pi^{2} X_{c}^{2}} \sin ^{2}\left(\pi B X_{c}\right) \sin \left(2 \pi X_{c} X_{0}\right)+E\left(X_{c}\right)\right] .
$$

By expanding $\cos \left(\eta_{1,2} X_{0}\right), \sin \left(\eta_{1,2} X_{0}\right), \cos \left(\frac{\pi}{2}\left(\eta_{2}^{2}-\eta_{1}^{2}\right)\right)$ and $\sin \left(\frac{\pi}{2}\left(\eta_{2}^{2}-\eta_{1}^{2}\right)\right)$ as power series in the relevant arguments it can be easily shown that the first term in the integral is of order $\frac{1}{\beta^{2}}$ and the second term is of order $\beta^{6}$ and higher order. By considering $\beta$ as a small parameter we take the first term to be our approximation to the asymmetry, interpret $E\left(X_{c}\right)$ as an error term and proceed to find bounds on its absolute value by finding bounds on the irtegrals $I_{1}, I_{3}$ and $I_{4}$.

Using the inequalities

$$
\begin{aligned}
|\sin (x)|,|\cos (x)| & \leq 1 \\
|\sin (x)| & <|x| \\
|\cos (x)-1| & <\frac{1}{2} x^{2}
\end{aligned}
$$


one can obtain upper bounds to the absolute values of the integrals $I_{1}$ and $I_{3}$ given by

$$
\begin{aligned}
\left|I_{1}\right| & \leq 2 \int_{-B}^{B} d \eta_{1} \int_{-B}^{B} d \eta_{2} \pi\left|\eta_{1}\right| X_{0} \frac{\pi}{2}\left|\eta_{2}^{2}-\eta_{1}^{2}\right| \\
& =\frac{11}{15} \pi^{2} X_{0} B^{5} \\
\left|I_{3}\right| & \leq \int_{-B}^{B} d \eta_{1} \int_{-B}^{B} d \eta_{2} \frac{1}{2}\left(\left(\pi \eta_{1} X_{0}\right)^{2}+\left(\pi \eta_{2} X_{0}\right)^{2}+\left(\frac{\pi}{2}\left(\eta_{2}^{2}-\eta_{1}^{2}\right)\right)^{2}\right) \\
& =\frac{4}{3} \pi^{2} X_{0}^{2} B^{4}+\frac{4}{45} \pi^{2} B^{6} \\
\left|I_{4}\right| & \leq \int_{-B}^{B} d \eta_{1} \int_{-B}^{B} d \eta_{2} \pi\left|\eta_{1}\right| X_{0} \pi\left|\eta_{2}\right| X_{0} \\
& =\pi^{2} X_{0}^{2} B^{4}
\end{aligned}
$$

Thus we obtain a bound to the modulus for the error term given by

$$
\pi^{2} \beta^{6} b^{4}\left(\frac{11}{15} x_{0} b+\frac{7}{3} x_{0}^{2}+\frac{4}{45} b^{2}\right)
$$

The error can clearly be seen to vanish when

$$
\beta=\sqrt{\frac{2}{\lambda} \frac{l+\bar{L}}{l L}} \rightarrow 0
$$

i.e. in the limit of infinitely long wavelengths and/or infinite system size, namely

$$
l, L \rightarrow \infty, \quad \frac{l}{L} \text { fixed }
$$

Notably this bound (2.39) is also significant for realistic experiments. Using the parameters from the two-slit experiment by Jönsson [14] (which incidentally did not include the AB effect), namely

$$
\begin{array}{r}
b=0.25 \mu \mathrm{m} \\
x_{0}=1 \mu \mathrm{m} \\
\lambda=5 \times 10^{-6} \mu \mathrm{m}
\end{array}
$$

and reasonable distances of

$$
\begin{aligned}
& l=10 \mathrm{~m} \\
& L=1 \mathrm{~m}
\end{aligned}
$$

the error bound is

$$
0.83 \times 10^{-2}
$$

compared with the peak value of

$$
0.104
$$

We thus expect the agreement between the exact diffraction pattern and our approximate form to be good. This is borne out by Figure 3 where the approximate and exact forms are in good agreement. Notably, if we increase $\lambda$ even by a single order of magnitude, the error bound drops by three orders of magnitude to

$$
0.83 \times 10^{-5}
$$

this time compared to a peak value only ten times less

$$
0.0108 \text {. }
$$

In this case there is no visible difference between the exact and approximate diffaction patterns (see Figure 4). (An equivalent effect could also be achieved by increasing the system size by a factor of 10 although this is difficult to realize experimentally.)

We note two important features of the approximate analytic form. The first is that $x_{c}^{2} A\left(x_{c}\right)$ is perfectly periodic in $x_{c}$ with period given as the lowest common multiple of

$$
\frac{2}{\lambda L} \frac{1}{b} \text { and } \frac{2}{\lambda L} \frac{1}{x_{0}}
$$

The second is that the dependence on $x_{c}$ only appears in the combination

$$
\beta^{2} \frac{l}{l+L} x_{c}=\frac{2 x_{c}}{\lambda L}
$$

Thus to lowest order in $\beta$ the whole diffraction pattern scales linearly with $L$, the distance between the screen with the slits and the observation screen. 


\section{EXPECTATION VALUES}

A. Normalisation of the wavefunction at the screen

Although the source wavefunction is a delta function and therefore cannot be normalized one can normalize the wavefunction at the screen, $\Psi^{\prime}\left(x_{c}\right)$. We proceed to determine bounds on this norm for the case of the flux parameter $\phi=\frac{\pi}{2}$ since this flux gives the greatest asymmetry in the diffraction pattern. Define

$$
\begin{aligned}
N\left(x_{c}\right) & =\left|\Psi^{\prime}\left(x_{c}\right)\right|^{2}+\left|\Psi^{\prime}\left(-x_{c}\right)\right|^{2} \\
& =2\left|\Psi_{+}\left(x_{c}\right)\right|^{2}+2\left|\Psi_{-}\left(x_{c}\right)\right|^{2}
\end{aligned}
$$

where $\Psi^{\prime}\left(x_{c}\right)$ and $\Psi_{+}\left(x_{c}\right)$ are defined in equations (2.16) and (2.2) respectively. Then the norm of the wavefunction is given by

$$
\mathcal{N}=\int_{0}^{\infty} d x_{c} N\left(x_{c}\right)
$$

Substituting the analytic expression for $\Psi_{+}\left(x_{c}\right)(2.8)$ and using the relation $\Psi_{-}\left(x_{c}\right)=$ $\Psi_{+}\left(-x_{c}\right)$ from $(2.14)$, one obtains

$$
\begin{aligned}
N\left(x_{c}\right) & =2 N^{2}\left[\left\{C\left(\beta\left(x_{0}+b-\frac{l}{l+L} x_{c}\right)\right)-C\left(\beta\left(x_{0}-b-\frac{l}{l+L} x_{c}\right)\right)\right\}^{2}\right. \\
& +\left\{S\left(\beta\left(x_{0}+b-\frac{l}{l+L} x_{c}\right)\right)-S\left(\beta\left(x_{0}-b-\frac{l}{l+L} x_{c}\right)\right)\right\}^{2} \\
& +\left\{C\left(\beta\left(x_{0}+b+\frac{l}{l+L} x_{c}\right)\right)-C\left(\beta\left(x_{0}-b+\frac{l}{l+L} x_{c}\right)\right)\right\}^{2} \\
& \left.+\left\{S\left(\beta\left(x_{0}+b+\frac{l}{l+L} x_{c}\right)\right) \cdots S\left(\beta\left(x_{0}-b+\frac{l}{l+L} x_{c}\right)\right)\right\}^{2}\right]
\end{aligned}
$$

where $N$ is given by (2.9). Again using the dimensionless constants defined in equations (2.24) - (2.26) and redefining the variables of integration so that the integration range runs from $-B$ to $B=\beta b$ one obtains

$N\left(x_{c}\right)=4 N^{2} \int_{-B}^{B} d \eta_{1} \int_{-B}^{B} d \eta_{2} \cos \left(\frac{\pi}{2}\left(\eta_{1}^{2}-\eta_{2}^{2}\right)\right) \mid \cos \left(\pi X_{0} \eta_{1}\right) \cos \left(\pi X_{0} \eta_{2}\right) \cos \left(\pi X_{c} \eta_{1}\right) \cos \left(\pi X_{c} \eta_{2}\right)$

$$
\begin{aligned}
& \left.+\sin \left(\pi X_{0} \eta_{1}\right) \sin \left(\pi X_{0} \eta_{2}\right) \sin \left(\pi X_{c} \eta_{1}\right) \sin \left(\pi X_{c} \eta_{2}\right)\right] \\
& =4 N^{2}\left[\int_{-B}^{B} d \eta_{1} \int_{-B}^{B} d \eta_{2} \cos \left(\pi X_{c} \eta_{1}\right) \cos \left(\pi X_{c} \eta_{2}\right)+I_{3}+I_{4}\right] \\
& =4 N^{2}\left[\frac{4}{\pi^{2} X_{c}^{2}} \sin ^{2}\left(\pi B X_{c}\right)+I_{3}+I_{4}\right]
\end{aligned}
$$

where first term is of order $\frac{1}{\beta^{2}}$ and the last two terms are of order $\beta^{6}$ and higher. The norm is then given as

$$
\begin{aligned}
& \mathcal{N}=4 N^{2}\left[\frac{4}{\pi^{2}} \int_{0}^{\infty} d x_{c} \frac{\sin ^{2}\left(\pi B X_{c}\right)}{X_{c}^{2}}+\int_{0}^{\infty} d x_{c}\left(I_{3}+I_{4}\right)\right] \\
& \hat{N}=4 N^{2}\left[2 \frac{l+L}{l} b+\frac{1}{\beta} \frac{L+l}{l} \int_{0}^{\infty} d X_{c}\left(I_{3}+I_{4}\right)\right]
\end{aligned}
$$

where the standard integral ( 15 ] 3.821.9) has been used. One now proceeds to find bounds on the absolute value of the second term in this equation. Firstly one must determine $x_{c}$ dependent bounds on the integrands $I_{3}$ and $I_{4}$. This is achieved by integrating the expressions (2.32) and (2.33) twice by parts one with respect to $\eta_{1}$ and once with respect to $\eta_{2}$ and again applying the inequalities (2.35) with the result that

$$
\left|I_{3}+I_{4}\right|<\frac{21 X_{0}^{2} B^{2}+4 B^{4}}{X_{c}^{2}}
$$

The range of the norm integral is then divided into two pieces. On the first interval $\left[0, \frac{4}{\pi B}\right]$ one uses the $x_{c}$ independent inequalities (2.37) and (2.38) to yield the following bound

$$
\begin{aligned}
\left|\int_{0}^{\frac{1}{\pi B}} d X_{c}\left(I_{3}+I_{4}\right)\right| & \leq \int_{0}^{\frac{4}{\pi B}} d X_{c}\left|I_{3}+I_{4}\right| \\
& \leq \int_{0}^{\frac{4}{\pi B}} d X_{c}\left(\left|I_{3}\right|+\left|I_{4}\right|\right) \\
& \leq\left(\frac{7}{3} \pi^{2} X_{0}^{2} B^{4}+\frac{4}{45} \pi^{2} B^{6}\right)\left(\frac{4}{\pi B}\right) \\
& =\pi B^{3}\left(\frac{28}{3} X_{0}^{2}+\frac{16}{45} B^{2}\right)
\end{aligned}
$$

On the second interval $\left[\frac{4}{\pi B}, \infty\right)$ one has

$$
\begin{aligned}
\left|\int_{\frac{1}{\pi B}}^{\infty} d X_{\mathrm{c}}\left(I_{3}+I_{4}\right)\right| & \leq \int_{\frac{1}{\pi B}}^{\infty} d X_{c}\left|I_{3}+I_{4}\right| \\
& <\int_{\frac{4}{\pi B}}^{\infty} d X_{c} \frac{21 X_{0}^{2} B^{2}+4 B^{4}}{X_{c}^{2}} \\
& =\pi B^{3}\left(\frac{21}{4} X_{0}^{2}+B^{2}\right)
\end{aligned}
$$


Combining equations (3.7) and (3.8) yields

$$
\left|\int_{0}^{\infty} d X_{c}\left(I_{3}+I_{4}\right)\right|<\pi B^{3}\left(14.584 X_{0}^{2}+1.356 B^{2}\right)
$$

Finally therefore

$$
\left|\mathcal{N}-8 N^{2} \frac{l+L}{l} b\right|<4 N^{2} \pi \frac{L+l}{l} \beta^{4} b^{3}\left(14.584 x_{0}^{2}+1.356 b^{2}\right)
$$

where the term of leading order in $\beta$ appears on the left hand side and the right hand side vanishes in the limit $\beta \rightarrow 0$. Using the parameters of reference [14] (2.42) - (2.43) one obtains

$$
(2.2-0.613) \mu \mathrm{m}<\frac{\mathcal{N}}{N^{2}}<(2.2+0.613) \mu \mathrm{m}
$$

It is also possible to calculate the norm using the full expression (3.4) and not just the leading order term. This has to be integrated numerically using either the series expansions or when appropriate the asymptotic expressions for the Fresnel integrals. If one integrates up to the three hundredth zero of $A\left(x_{c}\right)$ one obtains

$$
\frac{\mathcal{N}}{N^{2}}=2.194 \mu \mathrm{m}
$$

\section{B. Asymmetry parameter}

The asymmetry parameter which counts the number of electrons scattered to the right minus those scattered to the left is given by

$$
\begin{aligned}
A & \equiv \frac{1}{\mathcal{N}} \int_{0}^{\infty} d x_{c}\left|\Psi^{\prime}\left(x_{c}\right)\right|^{2}-\int_{-\infty}^{0} d x_{c}\left|\Psi^{\prime}\left(x_{c}\right)\right|^{2} \\
& =\frac{1}{\mathcal{N}} \int_{0}^{\infty} d x_{c} A\left(x_{c}\right)
\end{aligned}
$$

Evaluating this quantity to leading order in $\beta$ for flux parameter $\phi=\frac{\pi}{2}$ yields

$$
A=\frac{1}{2} \frac{1}{\beta b} \int_{0}^{\infty} d X_{c} \frac{4}{\pi^{2} X_{c}^{2}} \sin ^{2}\left(\pi B X_{c}\right) \sin \left(2 \pi X_{0} X_{c}\right)
$$

which can be evaluated using a standard integral ( [15] 3.763.3) to give

$$
A=\frac{1}{\pi} \vec{b}\left[\left(x_{0}+b\right) \ln \left(x_{0}+b\right)+\left(x_{0}-b\right) \ln \left(x_{0}-b\right)-2 x_{0} \ln \left(x_{0}\right)\right]
$$

This quantity is clearly non-zero as expected from the definition and the asymmetry of the diffraction pattern. Using the parameters of reference [14] (2.42-2.43), the quantity (3.15) becomes

\subsection{4}

compared with the value determined numerically using equation (2.28), which contains all contributions, instead of the leading contribution (3.15) of

$$
0.0772 \text {. }
$$

The numerical details are as discussed in the previous section.

\section{Expected displacement}

The average displacement, $\left\langle x_{c}\right\rangle$, is equal to

$$
\begin{aligned}
& \frac{1}{\mathcal{N}} \int_{-\infty}^{\infty} d x_{c} x_{c}\left|\Psi^{\prime}\left(x_{c}\right)\right|^{2} \\
& =\frac{1}{\mathcal{N}} \int_{0}^{\infty} d x_{c} x_{c} A\left(x_{c}\right)
\end{aligned}
$$

Evaluating this to leading order in $\beta$ yields

$$
\begin{aligned}
& \frac{1}{\mathcal{N}} 4 \sin \phi N^{2} \frac{1}{\beta^{2}} \frac{L+l}{l} \int_{0}^{\infty} d X_{c} \frac{4}{\pi^{2} X_{c}} \sin ^{2}\left(\pi B X_{c}\right) \sin \left(2 \pi X_{0} X_{c}\right) \\
& =0
\end{aligned}
$$

provided that the slits are disjoint i.e. $b<x_{0}$. Note that the standard integral ( [15] 3.763.2) has been used. One can also find a bound on the contribution to $\left\langle x_{c}\right\rangle$ from the error term $E\left(x_{c}\right)$, defined in Eq. (2.34). Firstly one integrates $I_{1}, I_{3}$ an $I_{4}$ by parts three times, twice on $\eta_{1}$ and once on $\eta_{2}$, and splits the integral $I_{3}-I_{4}$ as

$$
I_{3}-I_{4}=-\frac{4}{\left(\pi X_{c}\right)^{2}} \sin ^{2}\left(\pi B X_{0}\right)+I_{5}
$$


Then the same inequalitic: $(2.35)$ are used as previously to give bounds on the absolute values of $I_{1}$ and $I_{5}$ which do depend on $x_{c}$ and are given by

$$
\begin{aligned}
& \left|I_{1}\right|<\frac{59 X_{0} B^{2}}{\pi X_{c}^{3}} \\
& \left|I_{5}\right|<\frac{51 X_{0}^{2} B+15 B^{3}}{\pi X_{c}^{3}}
\end{aligned}
$$

Secondly the integral which gives the numerator for the average position is split into two intervals. On the first interval $\left[0, \frac{4}{\pi B}\right]$ one uses the $x_{c}$ independent inequalities (2.36) and (2.37) to obtain the following bound

$$
\begin{aligned}
\left|\int_{0}^{\frac{4}{\pi B}} d X_{c} X_{c} E\left(X_{c}\right)\right| & <\int_{0}^{\frac{4}{\pi B}} d X_{c} X_{c}\left|I_{1} \cos \left(2 \pi X_{0} X_{c}\right)+\left(I_{3}+I_{4}\right) \sin \left(2 \pi X_{0} X_{c}\right)\right| \\
& <\int_{0}^{\frac{4}{\pi B}} d X_{c} X_{c}\left(\left|I_{1}\right|+\left|I_{3}\right|+\left|I_{4}\right|\right) \\
& <\left(\frac{11}{15} \pi^{2} X_{0} B^{5}+\frac{7}{3} \pi^{2} X_{0}^{2} B^{4}+\frac{4}{45} \pi^{2} B^{6}\right) \frac{1}{2}\left(\frac{4}{\pi B}\right)^{2} \\
& =B^{2}\left(\frac{88}{15} X_{0} B+\frac{56}{3} X_{0}^{2}+\frac{32}{45} B^{2}\right)
\end{aligned}
$$

On the second interval $\left\{\frac{4}{\pi B}, \infty\right\}$ one first divides the integral using the split (3.20)

$$
\begin{aligned}
& \left|\int_{\frac{1}{\pi B}}^{\infty} d X_{c} X_{c} E\left(X_{c}\right)\right| \\
= & \left|\int_{\frac{1}{\pi B}}^{\infty} d X_{c} X_{c}\left(I_{1} \cos \left(2 \pi X_{0} X_{c}\right)+\left(I_{3}+I_{4}\right) \sin \left(2 \pi X_{c} X_{0}\right)\right)\right| \\
= & \left|\int_{\frac{1}{\pi}}^{\infty} d X_{c} X_{c}\left(I_{1} \cos \left(2 \pi X_{0} X_{c}\right)+\left[-\frac{4}{\left(\pi X_{c}\right)^{2}} \sin ^{2}\left(\pi B X_{0}\right)+I_{5}\right] \sin \left(2 \pi X_{c} X_{0}\right)\right)\right| \\
< & \left|\int_{\frac{4}{\pi B}}^{\infty} d X_{c} X_{c} \frac{4}{\left(\pi X_{c}\right)^{2}} \sin ^{2}\left(\pi B X_{0}\right) \sin \left(2 \pi X_{0} X_{c}\right)\right| \\
+ & \left|\int_{\frac{4}{\pi B}}^{\infty} d X_{c} X_{c}\left(I_{1} \cos \left(2 \pi X_{0} X_{c}\right)+I_{5} \sin \left(2 \pi X_{c} X_{0}\right)\right)\right|
\end{aligned}
$$

The first term in the rhs of equation(3.24) can be expressed in terms of a standard integral $([15] 8.230 .1)$ as

$$
\left|\frac{4}{\pi^{2}} \sin ^{2}\left(\pi B X_{0}\right) \operatorname{si}\left(8 \frac{X_{0}}{B}\right)\right|<4 B^{2} X_{0}^{2}\left|s i\left(8 \frac{X_{0}}{B}\right)\right|
$$

where si(a) denotes the sine integral

$$
s i(a)=-\int_{a}^{\infty} d t \frac{\sin t}{t}
$$

The second term in the rhs of equation (3.24) can be bounded using the $x_{c}$ dependent inequalities (3.21) and (3.22) to yield the following upper bound

$$
\begin{aligned}
& \left|\int_{\frac{4}{\pi B}}^{\infty} d X_{c} X_{c}\left(I_{1} \cos \left(2 \pi X_{0} X_{c}\right)+I_{5} \sin \left(2 \pi X_{c} X_{0}\right)\right)\right| \\
< & \int_{\frac{1}{\pi B}}^{\infty} d X_{c} X_{c}\left(\left|I_{1}\right|+\left|I_{5}\right|\right) \\
< & \int_{\frac{1}{\pi B}}^{\infty} d X_{c} X_{\mathrm{c}} \frac{1}{\pi X_{c}^{3}}\left(59 X_{0} B^{2}+51 X_{0}^{2} B+15 B^{3}\right) \\
= & B^{2} \frac{1}{4}\left(59 X_{1} B+51 X_{0}^{2}+15 B^{2}\right)
\end{aligned}
$$

Combining equations (3.23), (3.25) and (3.27) yields the following bound for the numerator of the average displacement

$$
\begin{aligned}
\left|\int_{0}^{\infty} d x_{c} x_{c} A\left(x_{c}\right)\right|< & 4 N^{2} \sin (\phi) \beta^{2} \frac{L+l}{l} b^{2} \\
& {\left[20.62 x_{0} b+\left(31.42+4\left|s i\left(\frac{8 x_{0}}{b}\right)\right|\right) x_{0}^{2}+4.462 b^{2}\right] }
\end{aligned}
$$

Combining this equation (3.28) together with the bound on the norm of the wavefunction (3.10) gives the final result for the upper bound of the average displacement for flux parameter $\phi=\frac{\pi}{2}$

$$
\begin{aligned}
\left|<x_{\mathrm{c}}>\right|< & {\left[8 N^{2} \frac{l+L}{l} b\left(1-\frac{\pi}{2} \beta^{4} b^{2}\left(14.584 x_{0}^{2}+1.356 b^{2}\right)\right)\right]^{-1} } \\
& 4 N^{2} \beta^{2} \frac{L+l}{l} b^{2}\left[20.62 x_{0} b+\left(31.42+4\left|s i\left(\frac{8 x_{0}}{b}\right)\right|\right) x_{0}^{2}+4.462 b^{2}\right] \\
= & \frac{1}{2} b\left[1-\frac{\pi}{2} \beta^{4} b^{2}\left(14.584 x_{0}^{2}+1.356 b^{2}\right)\right]^{-1} \\
& \beta^{2}\left[20.62 x_{0} b+\left(31.42+4\left|s i\left(\frac{8 x_{0}}{b}\right)\right|\right) x_{0}^{2}+4.462 b^{2}\right]
\end{aligned}
$$

This bound then vanishes in the limit $\beta \rightarrow 0$, i.e. in the limit of infinitely long wavelength and/or infinite system size meaning that the expected displacement in this limit is exactly zero. Since it seems reasonable to assume that the average displacement should increase as the distance between the screen with the slits and the observation screen increases, this 
result strongly suggests (although it in no way proves) that the average displacement will be zero in all cases. This result agrees with those obtained in references [4], [6]- [8] although the derivation is completely different. In particular our result is valid for all wavelengths and slit geometries but is only proven in the limit of infinite source-slit and slit-screen spacing The value of this bound for the experimental setup of Jönsson et al. [14] is

$$
\left|<x_{\mathrm{c}}>\right|<2.82 \mu \mathrm{m}
$$

It is also possible to obtain a bound for the parameters used by Kobe et al. [10], namely

$$
\begin{aligned}
\lambda & =1.74 \mathrm{~nm} \\
b & =1 \mathrm{~nm} \\
x_{0} & =3 \mathrm{~nm}
\end{aligned}
$$

This bound is given by

$$
\left|<x_{c}>\right|<2.2 \times 10^{-7} \mathrm{~nm}
$$

which clearly contradicts the numerical result in that work, the numerical discrepancy being too great to be explained by the difference between the Gaussian source in that work and the delta source used here. It is not however possible to comment meaningfully on the earlier calculation of Kobe [9] since in that caiculation Gaussian slits are used which then overlap. If in fact one calculates the average displacement for the case of overlapping rectangular slits i.e. $b>x_{0}$ then one obtains a non-zero contribution to the average position from the leading order term.

\section{CONCLUSIONS}

A leading order analytic expressions for the Aharonov-Bohm diffraction pattern has been obtained which is exact in the limit of long wavelengths and/or infinite source-slit-screen spacing and good for realistic experimental setups. Using this form one obtains a nonzero asymmetry for the number of electrons scattered but a zero value for their average displacement. If a non-zero value for $\left\langle x_{\mathrm{c}}\right\rangle$ is present, then it must be due to terms of higher order in $\beta$.

\section{ACKNOWLEDGMENTS}

VCAN thanks Prof. E. Friedland for his hospitality at the University of Pretoria. The financial support of the Foundation for Research Development, Pretoria, is also gratefully acknowledged. 


\section{REFERFNCES}

[1] Y. Aharonov and D. Bohm, Phys. Rev. 115, 485 (1959).

[2] M. Peshkin and A. Tonomura, The Aharonov-Bohm Effect, (New York: Springer-Verlag, 1989).

[3] T. H. Boyer, Phys. Rev. D 8, 1679 (1973).

[4] Y. Aharonov, C. K. Au, E. C. Lemer and J. Q. Liang, Lett. Nuovo Cim. 39, 145 (1984)

[5] S. Olariu and I. I. Popescu, Rev. Mod. Phys. 57, 339 (1985)

[6] M. D. Semon and I. R. Taylor, Il Nuovo Cim. 97, 25 (1987).

[7] M. D. Semon and J. R. Taylor, Il Nuovo Cim. 100, 389 (1987)

[8] M. D. Semon and J. R. Taylor, Found. of Phys. 18, 731 (1988).

[9] D. H. Kobe, Ann. Phys. (N.Y.) 123, 381 (1979).

[10] D. H. Kobe, V. C. Aguilera-Navarro and R. M. Ricotta, Phys. Rev. A 45, 6192 (1992)

[11] D. H. Kobe and V. C. Aguilera-Navarro, "Reply to Comment on 'Asymmetry of the Aharonov-Bohm diffraction pattern and Ehrenfest's theorem' ", submitted for publication.

(12) R. P. Feynman and A. R. Hibbs, Quantum Mechanics and Path Integrals (McGraw-Hill, New York, 1965)

[13] D. Shapiro and W. C. Henneberger, J. Phys. A: Math. Gen. 22, 3605 (1989)

[14] C. Jönsson, Zeitschrift fur Physik 161, 454 (1961)

[15] I. S. Gradshteyn and I. M. Rhyzik, Table of Integrals, Series and Products, Academic Press, New York (1980)
FIGURES

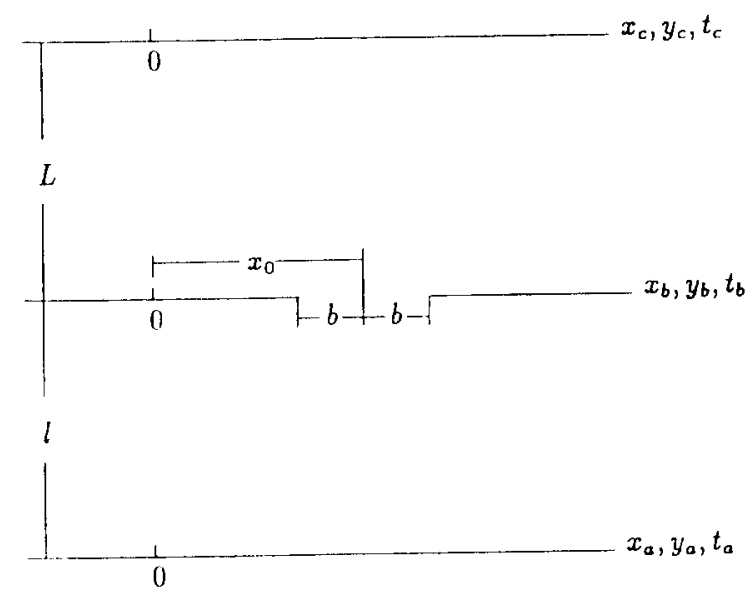

FIG. 1. The geometry of the single-slit diffraction experiment. The slit at $y_{b}$ has width $2 b$ and is centered at $x_{b}=x_{0}$. The distances $l$ and $L$ are given by equations (2.6) and (2.7) respectively.

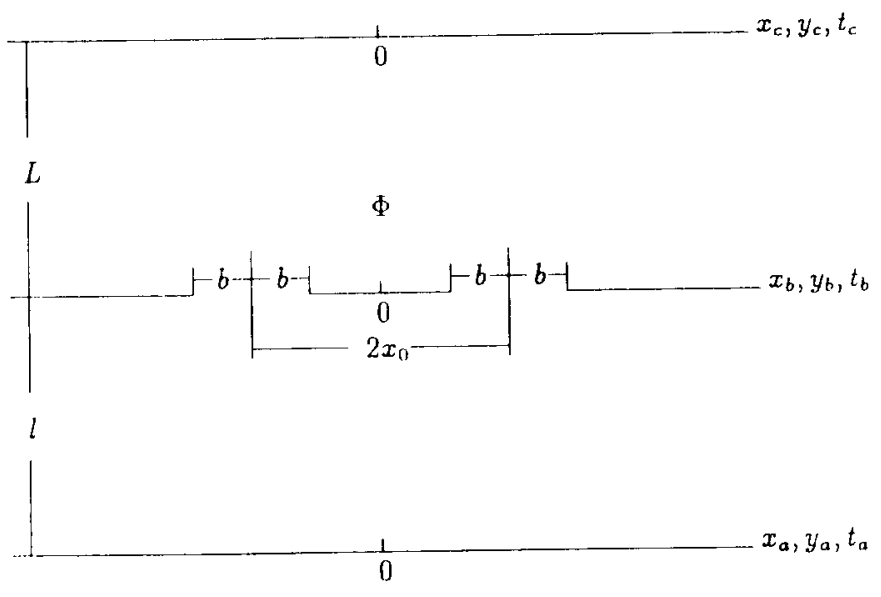

FIG. 2. The geometry of the two-slit diffraction experiment. Magnetic flux $\Phi$ is present in a shielded solenoid at $x_{b}=0$. The slits of width $2 b$ are centered at $\pm x_{0}$, and are separated by a distance $2 x_{0}$. The distances $l$ and $L$ are given by equations (2.6) and (2.7) respectively 


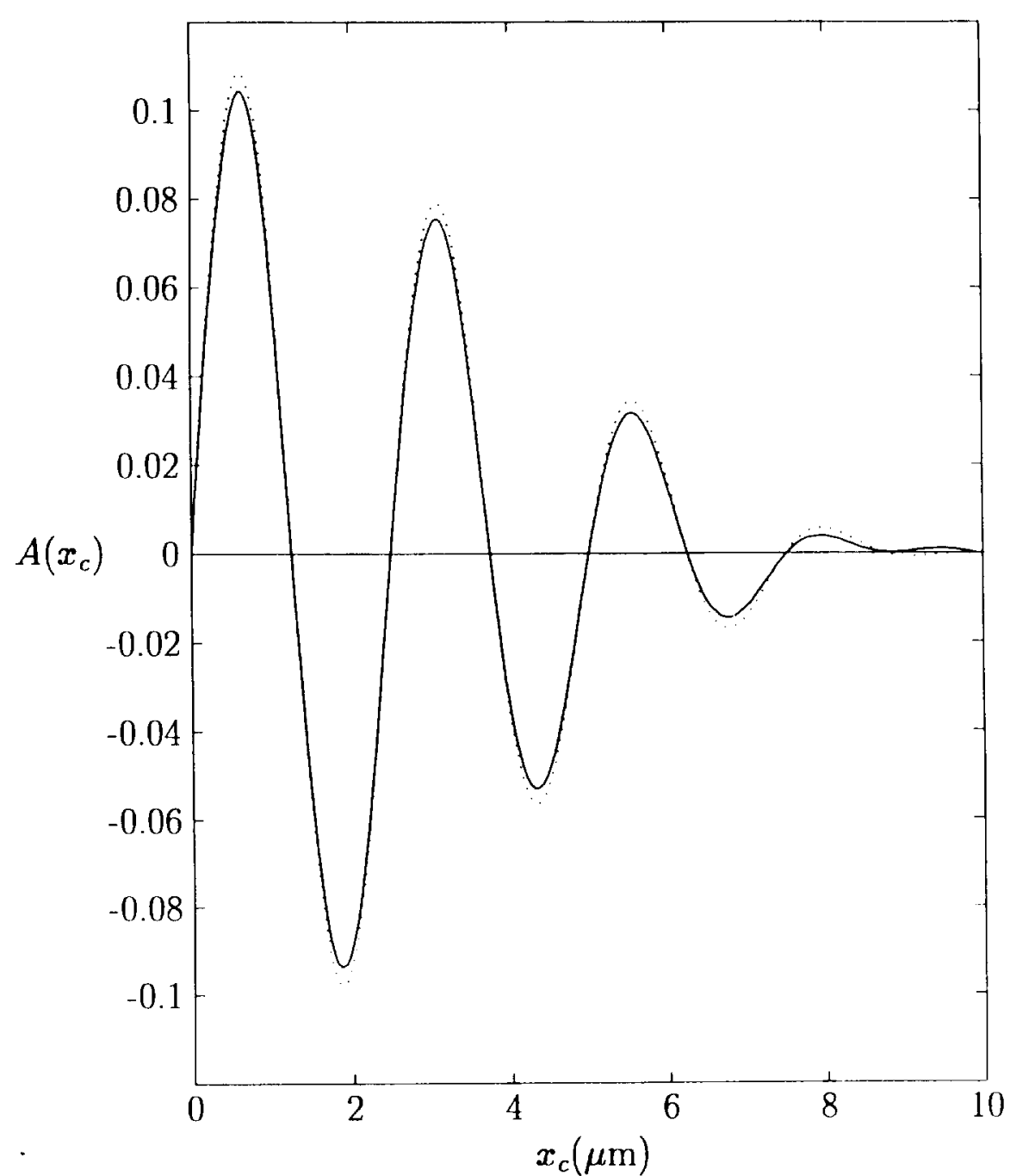

FIG. 3. The asymmetry in the two-slit diffraction pattern for electrons of wavelength $\lambda=5 \times 10^{-6} \mu \mathrm{m}$ with slit length $2 b=0.5 \mu \mathrm{m}$, slit spacing $2 x_{0}=2 \mu \mathrm{m}$, source - slit distance $l=10 \mathrm{~m}$, and slit to observation screen distance $L=1 \mathrm{~m}$. Since the wavefunction has not been normalised the scale on the $y$-axis is arbitrary. The solid curve shows the exact result and the dotted curve the approximate form.

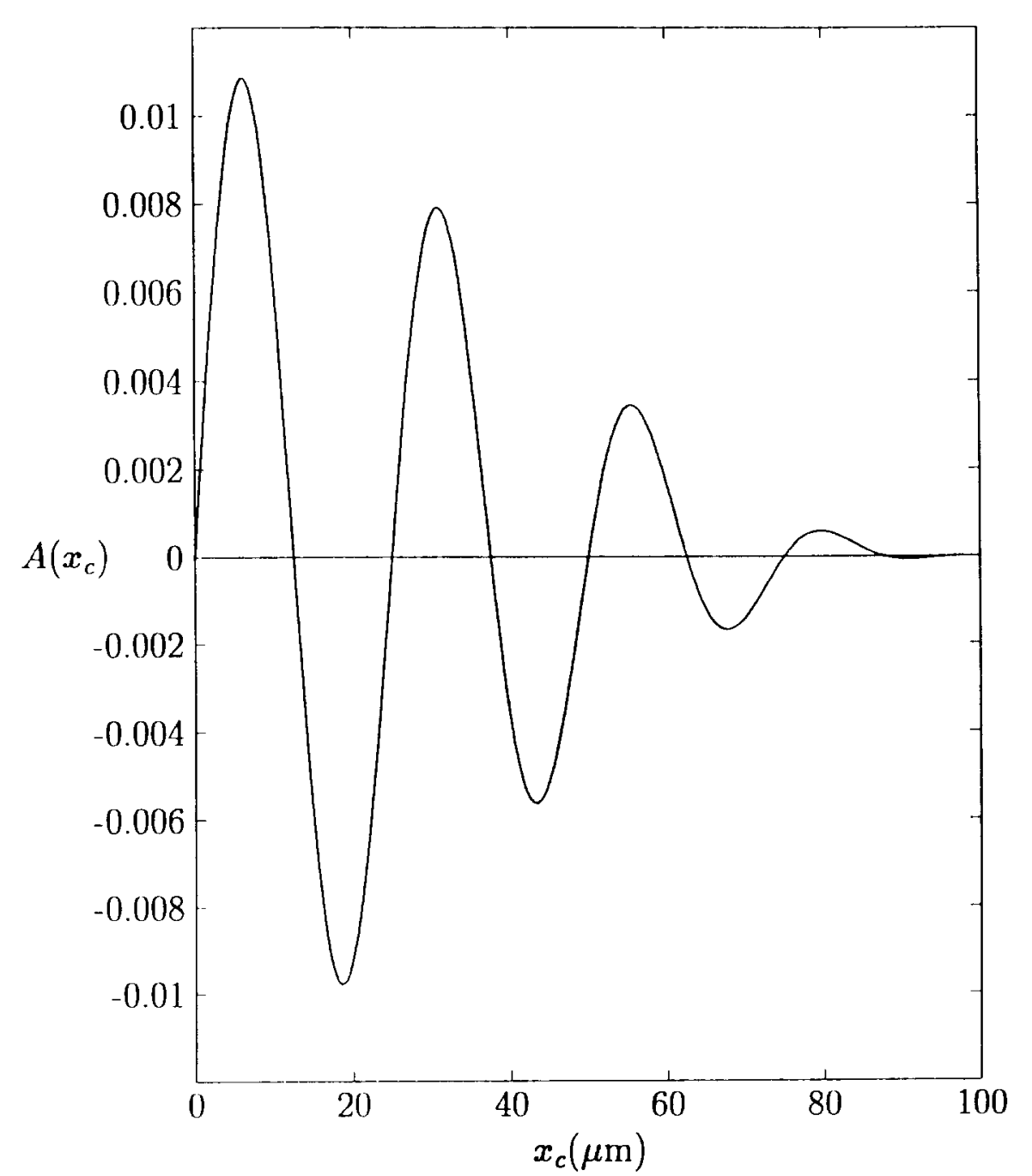

FIG. 4. The asymmetry in the two-slit diffraction pattern as in Figure 3 but with electron wavelength $\lambda=5 \times 10^{-5} \mu \mathrm{m}$. Here the approximate and the exact results coincide. 
